\title{
Cluster-Based Minimum Mean Square Estimation for Secure and Resilient Localization in Wireless Sensor Networks
}

\author{
Cliff Wang \\ U.S. Army Research Office \\ An Liu, Peng Ning \\ North Carolina State University
}

\begin{abstract}
Wireless sensor networks can support a wide variety of applications ranging from military surveillance to health care clinic monitoring. To carry out many of these tasks, a wireless sensor network must obtain accurate location for each sensor. A number of localization schemes have been developed to allow each sensor node to acquire its location. However, most of these techniques assume benign environments, and thus cannot survive malicious attacks in hostile environments where external and/or compromised nodes may launch attacks. This paper proposes a new computationally efficient and resilient localization scheme based the clustering of benign location reference anchors. Moreover, this paper reports both simulation and field experiments using a test-bed of MICAz motes performed to compare the proposed approach with several recent secure localization schemes. The experimental results demonstrate that the proposed scheme has the fastest execution time among all resilient localization schemes that can be used for the current generation of sensor platforms (e.g., MICA series of motes).
\end{abstract}

Keywords: Localization, wireless sensor networks, robustness and attack resiliency

\section{Introduction}

Wireless sensor networks (WSN) have found a great variety of applications ranging from health monitoring in the civilian world to military surveillance and reconnaissance. Location information is the key to many networking protocols such as geographical routing protocols (e.g., GPSR [10] and GEAR [29]) or geographic data-centric storage (e.g., GEM [21], GHT [25])) . Further, location information is needed to enable WSN applications such as target tracking (e.g., ZebraNet [8]) and environmental monitoring. To support these protocols and applications, nodes in WSN need to acquire and maintain accurate information of their locations.

The Global Positioning System (GPS) [28] is a popular outdoor localization system for mobile devices. However, due to the cost reasons, it is highly undesirable to have a GPS receiver on every sensor node. Moreover, in some situations such as indoor sensor network applications, GPS cannot be used for localization because of interferences and obstacles. This creates a demand for efficient and cost-effective location discovery algorithms in WSN.

There has been intensive investigation of localization techniques that do not entirely depend on GPS in recent years. Such techniques use some special nodes called anchors, which know their own locations (e.g., through GPS receivers or manual configuration), to help the other nodes discover locations. Existing localization schemes can be classified into range-based (e.g., [4, 19, 22, 26, 27]) or range-free techniques (e.g., [1, 5, 18, 23]). Range-based localization uses Received Signal Strength Indicator (RSSI), Time of Arrival (TOA), or Time Difference of Arrival (TDOA) to estimate the distance between the node that needs to discover its location and each reachable anchor, and estimates the node's location based on these distances and the anchors' locations. To reduce the demand on sophisticated hardware, computing power, and energy, range-free localization does not require physical distance measurement, but uses other means (e.g., centroid of all reachable anchors [1], overlap of triangles formed by reachable anchors [5], or hop counts from anchors [23]) for location estimation.

WSN may be deployed in hostile environments, and localization may become the target of attacks due to its importance. Most existing localization techniques assume benign environments and do not consider malicious attacks. Though we may use cryptographic message authentication to protect localization related messages, an attacker may easily bypass such protections by, for example, compromising unattended anchors. The threats to WSN localization in a hostile environment mandates the development of secure and resilient localization algorithms. Further, due to 
the resource constraints of a typical sensor node, the secure and resilient algorithm has to be efficient in terms of computation and memory requirement.

There have been recent developments of secure and resilient localization schemes for WSN [2,11-15]. AttackResistant Minimum Mean Square Estimation (ARMMSE) was first published in [15], and later an improved algorithm called Enhanced ARMMSE (EARMMSE) was developed in [14]. It is based on the observation that a location reference introduced by a malicious attack is usually "different" from benign ones, since it is aimed at misleading location estimation. Thus, ARMMSE uses the "inconsistency" among the location references provided by anchors to identify the malicious injection, and discard them before making final location estimation. The voting-based location estimation $[14,15]$ has each location reference "vote" on the locations at which the node of concern may reside. To facilitate the voting process, this approach partitions the deployment field into a grid of cells, and has each node determine how likely it is in each cell based on each location reference. It then selects the cell(s) with the highest vote and use the "center" of the cell(s) as the estimated location. Li et al. [13] studied and developed an attack resilient location estimator based on Least Median of Squares (LMS). The idea is to draw random subsets of data from the original data pool for individual subset estimation and then combine these estimates based on estimation quality. There are several other secure and resilient localization techniques, including SeRLoc [12], SPINE [2], and ROPE [11]. However, SeRLoc requires directional antenna on sensor nodes, SPINE requires nano-second scale time synchronization among sensor nodes, and ROPE, which is an integration of SeRLoc and SPINE, requires both directional antenna and nano-second scale time synchronization. These requirements cannot be met on the current generation of sensor platforms such as MICA series of motes.

Given the severe resource constraints (e.g., limited computing power, depleteable battery power) of the current generation of sensor platforms, a usable resilient localization scheme must be lightweight and computationally efficient. In this paper, we develop a novel computationally efficient and resilient localization scheme, which is more efficient than all the previous approaches with bounded location estimation error similar to the previous approaches. The computation efficiency and attack resiliency make the proposed approach suitable for resource constrained sensor nodes. Moreover, with the exception of $[14,15]$, all the other approaches have not been validated on real sensor platforms. To evaluate and compare the new algorithm with existing schemes, we implemented all the resilient localization schemes that can be used for the current generation of sensor platforms in TinyOS, and perform extensive evaluation and comparison of all these approaches through both simulation and experiments in a testbed of MICAz motes. Our experimental results demonstrate that the new approach proposed in this paper is fastest among all the ones evaluated.

The contribution of this paper is two-fold. First, we develop a novel, computationally efficient and resilient localization scheme for resource constrained sensor platforms. Second, we implement all the resilient localization schemes that can be used for the current generation of sensor platforms, and perform thorough experiments to compare their localization accuracy and efficiency in presence of malicious attacks. In particular, our implementation offers a readily available code base for integration into location-aware WSN applications.

The rest of this paper is organized as follows. The next section describes our assumptions and threat model. Section 3 discusses the cluster-based minimum mean square estimation (CMMSE) algorithm for secure and resilient localization in wireless sensor networks. Section 4 presents the implementation and the experiments of the proposed schemes as well as several other resilient localization schemes through both simulation and field experiments. Section 5 concludes this paper and points out some future research directions.

\section{Assumptions and Threat Model}

We first clarify our assumptions and threat model to facilitate the discussion. We assume that a WSN consists of a large number of regular sensor nodes (e.g., MICA motes) that need to estimate their locations and a small fraction of special anchors that are location aware (through, e.g., GPS receivers or manual configuration). We assume that the anchors are roughly uniformly distributed in the network, and each regular sensor node can obtain localization information from a sufficient number of anchors. For simplicity, we assume that a WSN operates on a 2-dimension plane, though the algorithms investigated in this paper can all be used for 3-dimension space with slight modification.

We focus on range-based localization in this paper. A regular node gets two pieces of information from each anchor that it communicates with for localization purpose: the location of the anchor and the distance between them. While the location of an anchor is usually provided by the anchor directly in a localization packet, there are multiple ways to obtain the distance between them, for example, using RSSI, ToA, or TDoA. We assume a sensor network may use any method to obtain these two pieces of information. Following [14,15], we abstract the localization information 
that a regular node obtains from an anchor as a location reference, represented as a triple $(x, y, d)$, where $(x, y)$ is the coordinate of the anchor, and $d$ is the estimated distance between these two nodes. We assume there may be errors in the estimated distances. However, when there is no malicious attacks, all distance measurement errors are bounded by $\epsilon_{\max }$, i.e., $-\epsilon_{\max }<\epsilon<\epsilon_{\max }$, where $\epsilon$ is any distance measurement error obtained in attack-free environments. Based on the location references received from multiple anchors, a regular sensor node runs a localization algorithm to estimate its own location.

We assume all localization related packets are authenticated (e.g., using TinySec [9]). Moreover, we assume each anchor is uniquely identified. This can be achieved with key management schemes that can provide unique pairwise keys for different pairs of nodes (e.g., TinyKeyMan [16, 17], random pairwise keys scheme [3]). Moreover, each regular node uses at most one location reference from each anchor. This is possible because each regular node can uniquely identify each anchor using the unique pairwise key shared between them.

We consider both external and insider attacks. When launching external attacks, the adversary does not control any valid node in the network. Though message authentication is effective in preventing the adversary from forging localization related packets, it cannot stop all external attacks. For example, the adversary may replay previously intercepted localization related packets captured at different locations. Moreover, the adversary may launch wormhole attacks [6] by creating low latency and high bandwidth communication channels between different locations in the network, faking non-existing links. The adversary may compromise anchors and launch insider attacks. The adversary may send incorrect locations, or manipulate the transmission of localization related packets (e.g., by using overly high or low transmission power if RSSI is used for distance measurement).

The adversary may jam the communication channel to launch Denial of Service (DoS) attacks. However, we assume that the adversary cannot constantly jam the communication channel without being detected and removed.

In both external and insider attacks, the adversary may convince regular sensor nodes to accept malicious location references. However, a regular node will accept at most one location reference from each compromised anchor or benign anchor whose localization packets are manipulated by the adversary, regardless how the adversary launches the attacks. Thus, in our security analysis, we only need to consider the location references that a regular node accepts, though there are many ways for the adversary to introduce malicious references. Finally, we assume there are more benign anchors than colluding malicious ones.

The primary objective of any secure and resilient localization scheme is to ensure that adversaries cannot introduce arbitrary localization errors under the above assumptions. Moreover, we would like to establish a good resiliency with the least computation overhead. In other words, we would like to develop light weight location estimation algorithms that can provide reasonably good location estimate with the presence of injected errors from malicious anchors.

\section{Cluster-Based Minimum Mean Square Estimation (CMMSE)}

In this section, we present a new secure and resilient localization scheme, called Cluster-Based Minimum Mean Square Estimation (CMMSE). This approach achieves higher efficiency than existing approaches while providing comparable resilience against malicious location references. Similar to a previous work, ARMMSE [15], CMMSE is based on the Minimum Mean Square Estimation (MMSE) method proposed in [26]. In the following, we first give a brief overview of the basic MMSE method, and then present our new approach.

\subsection{Basic MMSE for WSN Localization [26]}

Assume that a regular sensor node has obtained a set of $k$ location references from the reachable anchors, $\left\{\left(x_{0}, y_{0}, d_{0}\right)\right.$, $\left.\left(x_{1}, y_{1}, d_{1}\right), \ldots,\left(x_{k-1}, y_{k-1}, d_{k-1}\right)\right\}$. Suppose the estimated location is $(\hat{x}, \hat{y})$. Thus, the error of the measured distance between the regular node and the $i$ th $(0 \leq i \leq k-1)$ anchor can be expressed as the difference between the measured distance $d_{i}$ and the estimated distance, i.e., $e_{i}=d_{i}-\sqrt{\left(\hat{x}-x_{i}\right)^{2}+\left(\hat{y}-y_{i}\right)^{2}}$.

The basic MMSE method obtains the location estimate $(\hat{x}, \hat{y})$ by minimizing the mean square error (MSE)

$$
M S E=\frac{1}{k} \sum_{i=0}^{k-1}\left[\sqrt{\left(\hat{x}-x_{i}\right)^{2}+\left(\hat{y}-y_{i}\right)^{2}}-d_{i}\right]^{2} .
$$

Before we present our algorithm, we first obtain a property of the basic MMSE method when there is no malicious location reference. We will use this property in the later discussion. 


\subsubsection{Bound of Minimum MSE in Normal Situations}

As we discussed in Section 2, we assume a distance measurement error is bounded by $\epsilon_{\max }$. In the following, we show that the location estimation error is also bounded in such cases.

Lemma 1 Assume a regular node at $(x, y)$ receives a set of $k$ location references $\left\{\left(x_{0}, y_{0}, d_{0}\right), \ldots,\left(x_{k-1}, y_{k-1}, d_{k-1}\right)\right\}$. If all the location references are benign and the maximum distance measurement error for each location reference is bounded by $\epsilon_{\max }$, then the minimum MSE for the location estimate $(\hat{x}, \hat{y})$ obtained with MMSE is bounded by $\epsilon_{\text {max }}^{2}$.

Proof: Since distance measurement error is bounded by $\epsilon_{\max }$, we have

$$
\left|d_{i}-\sqrt{\left(x-x_{i}\right)^{2}+\left(y-y_{i}\right)^{2}}\right| \leq \epsilon_{\max }
$$

This implies

$$
\left(d_{i}-\sqrt{\left(x-x_{i}\right)^{2}+\left(y-y_{i}\right)^{2}}\right)^{2} \leq \epsilon_{\max }^{2}
$$

and

$$
\frac{1}{k} \sum_{i=1}^{k}\left(d_{i}-\sqrt{\left(x-x_{i}\right)^{2}+\left(y-y_{i}\right)^{2}}\right)^{2} \leq \epsilon_{\max }^{2} .
$$

Since an MMSE method produces a location estimate $(\hat{x}, \hat{y})$ that minimizes the MSE, we have

$$
\begin{gathered}
\frac{1}{k} \sum_{i=1}^{k}\left(d_{i}-\sqrt{\left(\hat{x}-x_{i}\right)^{2}+\left(\hat{y}-y_{i}\right)^{2}}\right)^{2} \\
\leq \frac{1}{k} \sum_{i=1}^{k}\left(d_{i}-\sqrt{\left(x-x_{i}\right)^{2}+\left(y-y_{i}\right)^{2}}\right)^{2} \leq \epsilon_{\max }^{2}
\end{gathered}
$$

To accommodate the limited computational power of current generation of sensor nodes (e.g., MICAz), the basic MMSE method proposed in [26] uses an approximate approach to estimating the location $(\hat{x}, \hat{y})$ [26]. This MMSE method is used in the work of ARMMSE [15] and we adopts the same method in this paper. However, when such an approximate method is used for MMSE calculation, the minimum MSE obtained from purely benign location references could be greater than $\epsilon_{\max }^{2}$ when the anchors providing the references are approximately located on a straight line (collinear case). Though there is no attack, such approximation method results in similar impact to malicious attacks, and thus is not desirable. Our algorithm will address such potentially large MMSE errors due to approximation using an optional consistent set expansion phase (Section 3.2.1).

\subsection{CMMSE: Achieving High Efficiency}

A malicious anchor can provide an arbitrary location reference by either changing its declared location $(x, y)$ or manipulate the distance measurement (e.g., by changing the transmission power if RSSI is used). When both benign and malicious location references co-exist and the malicious nodes inject arbitrary location reference errors, the minimum MSE obtained with an MMSE method will exceed the normal MSE bound, giving us an opportunity to discover and discard malicious location references.

Obviously the basic MMSE method cannot deal with malicious location references, since it can't distinguish and discard faulty location references from malicious nodes. A resilient algorithm needs to filter out malicious location references and uses the good location references to perform localization.

Based on the assumption of majority benign anchor nodes, the ARMMSE approaches developed in [14, 15] run multiple rounds of basic MMSE operations to search for the largest consistent benign location reference set. The ARMMSE approaches start from the whole set of location references, and runs MMSE based consistency check to filter out malicious ones iteratively. This is inefficient since MMSE calculation involves many matrix operations. The larger the number of location references, the more costly the MMSE calculation. 
In this paper, based on the same assumption of majority benign location references, we develop the cluster-based MMSE (CMMSE) method, aiming at achieving a higher computational efficiency than the ARMMSE methods. Our scheme takes an opposite approach by growing the largest consistent set from two randomly chosen location references (as the seed). If the two seeds selected are benign, we can grow the largest consistent location reference set within one round by checking each remaining location reference, using the MMSE threshold. The efficiency of this scheme depends on how quickly two benign location references can be found. When the percentage of benign ones is high, two benign seeds can be chosen in just a few rounds. Another important feature of this algorithm is that any MMSE calculation is only performed on three location references. As a result, the calculation can be performed very quickly. Furthermore, we develop a variation of the cluster-based algorithm, which can provide location estimation once more than half of benign location references are found. This provides further speedup of the algorithm. Section 3.3 provides a detailed analysis on the performance of this new approach.

\subsubsection{Algorithm}

Our CMMSE algorithm is based on the examination of location reference triplets. Given $k$ location references, there are a total of $\left(\begin{array}{l}k \\ 3\end{array}\right)$ possible triplets (of location references) that can be formed. If there are no malicious location references, any one of the $\left(\begin{array}{l}k \\ 3\end{array}\right)$ triplets may provide a good location estimate. However, due to malicious attacks, a triplet may have 0 to 3 malicious location references. It is obvious to see that with a total of $k$ location references and $m$ malicious ones, $\left(\begin{array}{c}k-m \\ 3\end{array}\right)$ triplets are free of malicious location references, and $\left(\begin{array}{c}m \\ 3\end{array}\right)$ triplets contain malicious location references only. The rest of the $\left(\begin{array}{l}k \\ 3\end{array}\right)-\left(\begin{array}{c}m \\ 3\end{array}\right)-\left(\begin{array}{c}k-m \\ 3\end{array}\right)$ triplets may contain one or two malicious location references each.

Each triplet of location references provides a location estimate $(\hat{x}, \hat{y})$ and a corresponding minimum MSE. In the case of a benign triplet, the minimum MSE is bounded by $\epsilon_{\text {max }}^{2}$, as shown in Lemma 1. (Note that when an approximated MMSE method such as [26] is used, a set of three approximately co-linear location references will lead to large minimum MSE. We will address this problem with an expansion phase, as discussed later.) If all three location references in a triplet are malicious and colluding with each other, the minimum MSE may also be bounded. In both cases, the minimum MSE is bounded because of the consistency of the three location references. However, when a triplet has both benign and malicious location references, or non-colluding malicious location references, the minimum MSE is no longer bounded, and can be arbitrarily large due to the injected error.

Based on the above intuition, we propose a lightweight, secure and resilient localization scheme, which we refer to as Cluster-Based MMSE (CMMSE). This scheme uses a simple MMSE threshold test to identify consistent triplets, and to form a consistent reference set for the final location estimation.

The algorithm runs in rounds. In each round, we first perform a triplet formation and examination phase to identify the consistent set of location references. We use the basic MMSE method [26] to perform location estimation. As discussed earlier, the minimum MSE of a benign triplet may be greater than the MSE bound due to the algorithm's approximation error when the three reference anchors are close to be collinear. To address this issue, a second expansion phase may be used to examine and include those benign location references removed incorrectly in phase I.

For the sake of presentation, we denote the set of all input location references as $S$, and the set of location references to use for the final location estimation as $C$.

\section{Phase I: Partitioning $S$ via Triplet Formation and Examination.}

1. Set two sets $C$ and $L$ both as empty sets.

2. We randomly select two location references $r_{1}$ and $r_{2}$ as the seeds, and perform a proximity check as follows: Assuming that $d_{i}$ and $d_{j}$ are the measured distances from the node to be localized to the two anchors and that the maximum radio signal range is $d_{\max }$, if $d_{i}+d_{j}<2 *\left(d_{\max }+\epsilon_{\max }\right), r_{1}$ and $r_{2}$ are accepted as seeds and put into $C$. Otherwise, reject these two seeds and repeat this step.

The rationale for this check is that two benign anchors from which a regular node receives localization references cannot be more than $2 d_{\max }$ away from each other.

3. Using the two seeds $r_{1}$ and $r_{2}$, we examine each of the remaining location references in $S$ one by one. Specifically, for each remaining location reference $r$ in $S$, it forms a triplet along with the two seeds $r_{1}$ and $r_{2}$. Following the basic MMSE method [26], we calculate the location estimation and its corresponding minimum MSE. If the minimum MSE is smaller than $\epsilon_{\max }^{2}$, the three anchors are consistent, and we put $r$ into the consistent set $C$. Otherwise, it is not consistent with the two seeds and is placed in the set $L$. 
After completing the above steps in Phase I, we split the original set $S$ of location references into the consistent set $C$ containing the two seeds and all nodes consistent with them, and the leftover set $L=S$ inconsistent with the seeds.

Phase II: Expansion of Consistent Set. During phase I, a location reference is placed into the leftover set $L$ when the minimum MSE is greater than the bound $\epsilon_{\max }^{2}$. As mentioned earlier, the basic MMSE method may generate large calculation error when the three corresponding anchors are approximately collinear. In the expansion phase, each location reference in the leftover set $L$ is checked against the whole reference set $C$ to see if these $|C|+1$ location references will generate a minimum MSE lower than $\epsilon_{\max }^{2}$. If yes, it is added into $C$. Any benign location reference mistakenly rejected in phase I due to the approximate error can be recovered.

After the execution of these two phases in one round, there are two possible outcomes:

1. $|C|>\frac{k}{2}$. In this case, there are $C$ has more than $\frac{k}{2}$ consistent location references. Under the assumption that there are more benign location references than the malicious ones, we have obtained the benign set and can use it to perform the final location estimate.

2. $|C| \leq \frac{k}{2}$. In this case, we fail to grow a consistent set larger than $\frac{k}{2}$. We then remove the two seeds $r_{1}$ and $r_{2}$ from $S$, since they cannot be used to form a consistent majority from the input location references. If $|S|>\frac{k}{2}$, we start a new round by repeating Phase I and Phase II. Otherwise, there is no chance to form a consistent majority based on the input, and thus the algorithm terminates.

Quick CMMSE (QCMMSE): A Variation. When all the location references used for the final location estimation are benign, the more location references we have, the more accuracy the final estimation can potentially be [26]. Thus, when accuracy is the highest priority, we should follow both phases in the above algorithm to get as many consistent location references as possible, even the consistent ones already form the majority of the input. However, when the execution time and the energy consumption is the highest priority, we may want to stop examining the remaining location references once we have obtained more than $\frac{k}{2}$ consistent location references, where $k$ is the total number of input location references. This leads to the QCMMSE variation of the proposed scheme. Specifically, we keep track of the size of $C$ in both Phase I and Phase II. We terminate the algorithm whenever $C$ has $\max \left\{3,\left\lfloor\frac{k}{2}+1\right\rfloor\right\}$ consistent location references, and then use $C$ for the final location estimation. This variation can effectively reduce the computation required by CMMSE, with a trade-off of slightly increased location estimation error.

\subsection{Complexity}

Each round of CMMSE is of complexity $O(k)$, given $k$ location references. We only need to run one round if the first two seeds selected are benign. Otherwise, multiple rounds are needed until two benign seeds are selected. Obviously, the number of rounds required depends on the number of malicious location references. A malicious location reference is in general not consistent with a benign one, and will not be selected as a seed along with a benign one. Thus, for $m$ malicious location references, at most $\left\lfloor\frac{m}{2}+1\right\rfloor$ rounds are needed in the worst case when each round selects two colluding malicious location reference as the seeds. This is possible probabilistically, but highly unlikely.

We can derive the probability of correctly selecting two benign seeds at round $i$ as follows:

$$
p(1)=\frac{(k-m) \times(k-m-1)}{k \times(k-1)},
$$

and

$$
p(i)=\frac{(k-m-(i-1)) \times(k-m-(i-1)-1)}{(k-2 \times(i-1)) \times(k-2 \times(i-1)-1)} \times \prod_{j=1}^{i-1}\left(1-\frac{(k-m-(j-1)) \times(k-m-(j-1)-1)}{(k-2 \times(j-1)) \times(k-2 \times(j-1)-1)}\right),
$$

when $i=2,3, \ldots,\left\lfloor\frac{m}{2}+1\right\rfloor$. In this equation for $p(i)\left(i=2,3, \ldots,\left\lfloor\frac{m}{2}+1\right\rfloor\right)$, the first part represents the probability of picking two benign location references in round $i$, and the second part represents the probability of not being able to pick two benign seeds in the previous rounds. The average number of rounds required to find the complete set can be found as $\sum_{i=1}^{\left\lfloor\frac{m}{2}+1\right\rfloor} p(i) \times i$. Figure 1 shows the average number of rounds in CMMSE in a few possible settings.

The average round required depends on the number of malicious location references. For example, in figure 1, when $k=19, m=3$, the average number of rounds is 1.16 . When $m$ increases to 9 , the average number of rounds 1.91. When there is no or a few malicious location references, CMMSE can finish fairly quickly. The QCMMSE variation performs less computation, and thus can finish more quickly than CMMSE. 


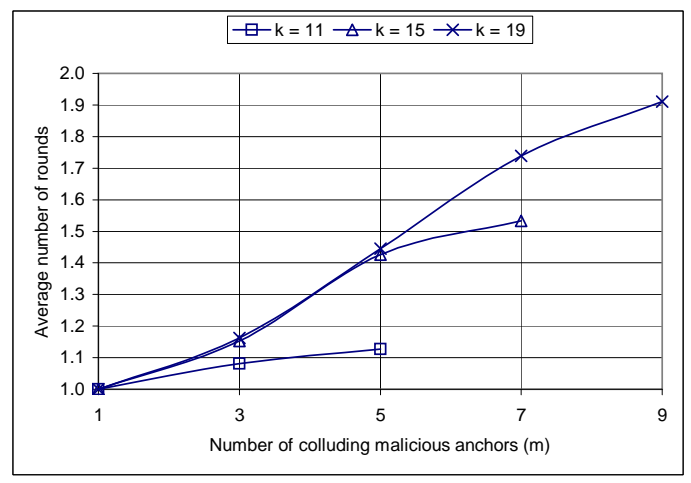

Figure 1: Average number of rounds in CMMSE in typical settings

\subsection{Security Analysis}

In this section we provide the security analysis of our localization scheme. We show that our scheme can tolerate arbitrary false messages introduced by compromised anchors as long as the benign anchors constitute the majority of all reachable anchors.

Based on our assumption that the location reference messages are cryptographically protected, an adversary cannot launch attacks simply by forging or modifying location reference messages without the knowledge of correct cryptographic keys. The adversary only has two ways to launch attacks: replay location reference messages captured in other places, and send malicious location reference messages through compromised anchors. The first case is indeed equivalent to a compromised anchor claiming to be in the same location as the original transmission location of the captured location reference message. Thus, we will focus our attention on attacks launched from compromised anchors. In this case, once the adversary has access to the keys on a compromised anchor, arbitrary but legitimate messages (that can be verified cryptographically) can be sent. Nevertheless, because of the unique pairwise key shared between any two nodes, each node will accept at most one location reference from each (potentially compromised) anchor.

The security of CMMSE follows from Lemma 1. When the injected location reference error is larger than the normal measurement error bound, the resulting minimum MSE will not be bounded by the threshold $\epsilon_{\max }$. This allows a normal sensor node to discover the malicious location references. As mentioned in Section 3.2.1, once two benign location references have been selected, we perform MMSE check on each triplet to filter out malicious location reference.

Although individual non-colluding malicious reference can be detected and removed easily, it is much harder to deal with colluding references. If two colluding location references are selected to be the triplet seeds, they will eventually be removed since they cannot be used to grow a majority consistent set. However, in this case, a regular sensor node has to run a full round of triplet examination before discarding the two colluding location references. Nevertheless, as discussed in Section 3.3, when the percentage of malicious anchors is low, the probability of selecting two malicious references as triplet seeds is low.

Similar to all previous work [2,11-15], our scheme relies on the assumption that the majority of the location references are benign. The adversary has to either increase the number of colluding malicious nodes or reduce the number of benign nodes in order to sabotage our localization scheme. For example, the adversary may jam the message sent by benign anchors. This can effectively reduce the set of benign location references. When the malicious colluding set has more nodes than the benign set, our algorithm fails. Dealing with physical layer attacks such as jamming or MAC layer denial of service attacks is outside the scope of this paper. There are techniques such as spread spectrum communication, special coding, and frequency hopping that can provide an efficient mechanism to shield the physical layer against jamming attacks.

Other possible attacks include wormhole attack [6] and Sybil attack [20]. We discuss them next.

Wormhole Attack: A wormhole is a direct tunnel between two points in the network established by the adversary [7]. Under normal network operations, there is no direct link between these two points due to the communication range or other constraints. The direct wormhole link is established by the adversary with the intention of eavesdropping and recording messages at one end (origin) of the wormhole link and replaying them at the other end (destination).

The wormhole attack can be launched against our localization scheme, but it will not be effective. When a remote 
location reference beacon is replayed at local neighborhood, the location coordinates $(x, y)$ in the replayed message reflects the original anchor location. The attacker may arbitrarily set the beacon signal strength to manipulate the distance measurement. But the distance manipulation is limited by the maximum anchor transmission range, and thus may not be consistent with the original anchor coordinates. (Indeed, as pointed out in [15], if the manipulated location reference is consistent with the other benign ones, it will not introduce localization error.) In addition, the replayed anchor coordinates also conflict with the local anchor coordinates (since they are not supposed to be within the same communication range to the node to be localized). As a result, the minimum MSE will exceed the measurement error bound, and the malicious location reference will be discarded.

For multiple remote location references to form a consistent set, the adversary not only has to replay these reference messages, but also has to replay them at different local locations mirroring the same geographic layout at the origin site. Otherwise, the replayed remote messages cannot form a complete consistent set and will be detected. Thus, a simple wormhole link can not effectively disrupt our localization scheme.

Sybil Attack: The adversary can launch a Sybil attack when it has compromised anchor nodes and cloned the victim anchors at different sites of a wireless sensor network [20]. In a Sybil attack, the attacker has access to the cryptographic keys on the compromised anchors and can use the same key in the cloned nodes. As a result, the attacker can distribute arbitrary information using valid node ID and keys and will not be detected by authentication check. The Sybil attack poses a great threat to our scheme since cloned nodes may invalidate our assumption of majority benign anchors.

To defeat Sybil attack, we need to detect and identify nodes that are cloned. A solution to detect the Sybil attack was recently proposed in [24], which relies on a third party to witness duplicated identity among the cloned nodes. In our scheme, assuming that that each anchor node shares a pairwise key with the base station, we can rely on the base station to detect cloning. Each anchor sends back to the base station a cryptographically protected message reporting all heard location references from other anchor nodes. Since the base station has the knowledge of each anchor node's deployment location, it can detect Sybil attack when location conflicts among the reports are discovered. The base station can then flood the whole network to revoke the cloned anchors.

\section{Implementation and Experimental Evaluation}

In this section, we report the implementation of the proposed schemes, as well as the experimental evaluation performed to compare the proposed schemes with all the secure and resilient localization techniques that can be used on the current generation of sensor platforms. In our evaluation, we first perform outdoor field experiments to understand the performance of the proposed schemes in a particular deployment, and then perform a large number of simulation experiments to obtain the performance results in general cases.

\subsection{Implementation}

To facilitate the experimental evaluation and comparison with the previous approaches, we implemented the proposed CMMSE and QCMMSE schemes as well as all recent secure and resilient localization techniques that can be used on the current generation of sensor platforms (e.g., MICA series of motes) running TinyOS, including EARMMSE [14], voting-based scheme [14], and LMS-based scheme [13].

Given a maximum number of location references, several schemes under investigation require a few parameters to be configured. These parameters must be set appropriately to ensure a fair comparison. For the EARMMSE scheme, we set the mean square error threshold $\tau=0.8 \epsilon_{\max }$ as discussed in [14], where $\epsilon_{\max }$ is the maximum distance measurement error. Based on our measurement in the field experiments, we set $\epsilon_{\max }=7.4 \mathrm{feet}$ and $\tau=$ $0.8 \epsilon=5.92$ feet. The critical parameter for the voting-based scheme is the number of cells $M$ in the grid in each iteration [14]. The cell number needs be the square of an integer. The voting-based scheme is in general much slower than CMMSE and QCMMSE, no matter how we configure $M$. To ensure a fair comparison, we use two instances of the voting-based scheme with $M=100$ and $M=225$, respectively, representing two distinct trade-offs between location estimation error and efficiency. Similar to the voting-based scheme, the LMS scheme [13] is much slower than the proposed schemes. To ensure fair comparison, we set the subset size $n=4$. Moreover, we set the number $M$ of subsets to be examined in such a way that the LMS scheme has the same average execution time as the voting-based scheme when the grid size is 100 and 225 , respectively, to match the above two instances of the voting-based scheme. 


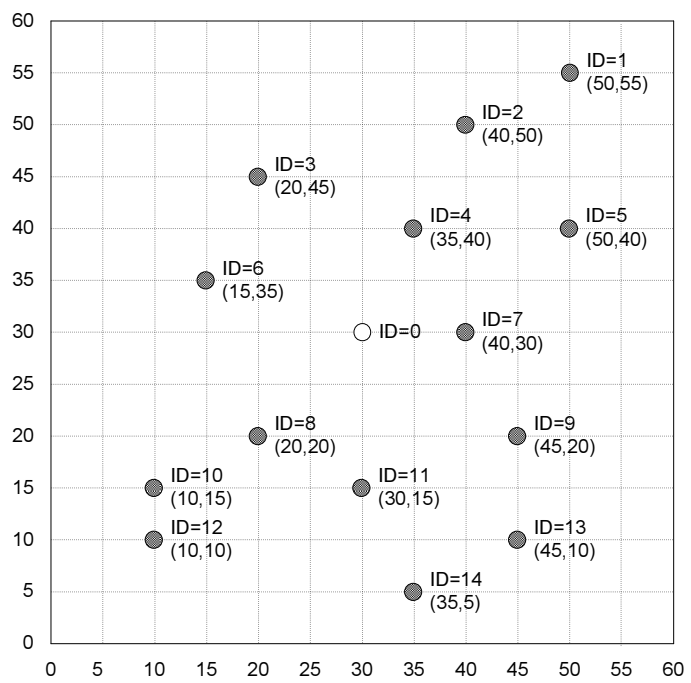

Figure 2: Nodes deployment in the field experiments (Test field size: $60 \mathrm{feet} \times 60 \mathrm{feet}$ )

Table 1 shows the code sizes of these schemes compiled for MICAz motes, assuming that each node uses up to 14 anchors. These numbers are obtained using the check_size.pl script provided in the TinyOS distribution. Note that the RAM size does not include the memory consumed by local variables in stack. Since the nesC Compiler optimizes the whole program by default, to compare the size of code fairly, we disabled the optimization of nesC compiler.

\begin{tabular}{|c|c|c|}
\hline Scheme & ROM (bytes) & RAM (bytes) \\
\hline Basic MMSE & 2,734 & 0 \\
\hline EARMMSE & 5,146 & 248 \\
\hline Voting (M=100) & 7,074 & 100 \\
\hline Voting $(\mathrm{M}=256)$ & 7,074 & 256 \\
\hline LMS & 5,262 & 237 \\
\hline CMMSE & 8,058 & 276 \\
\hline QCMMSE & 8,090 & 276 \\
\hline
\end{tabular}

Table 1: Code sizes of secure and resilient localization schemes under comparison (up to 14 location references)

\subsection{Field Experiments}

We perform a series of outdoor field experiments using MICAz motes to compare the proposed schemes with the other alternatives under investigation. These field experiments offer an opportunity to observe their performance in a realistic setting. We use the RSSI method to measure the distance, since this is the only option for MICAz motes.

In the outdoor field experiments, we deploy $15 \mathrm{MICAz}$ motes in a 60 feet $\times 60$ feet target field, as shown in figure 2. We use 14 motes as anchors to replicate a dense deployment. The anchors broadcast location reference messages periodically. The sensor node with ID 0 (in the middle of the field) is a regular node that needs to estimate its own location.

With this deployment setup, we perform experiments under four attack scenarios. In the first scenario, one randomly selected anchor is configured as being malicious, which reports a faulty location reference $x$ feet away from its true location in a random direction. In the second scenario, we randomly select four malicious anchors. Each malicious anchor adds a random location offset of $x$ feet from its true location. The third scenario mimics node collusion. Four randomly selected anchors collude with each other and send out false but consistent location references. In this case, all malicious anchors report a falsified position shifted $x$ feet from its true location in the same direction. In the fourth scenario, we experiment with a varying number of colluding anchors ranging from 1 to 8 (out of 14 anchors) to examine the impact on the estimated location. 


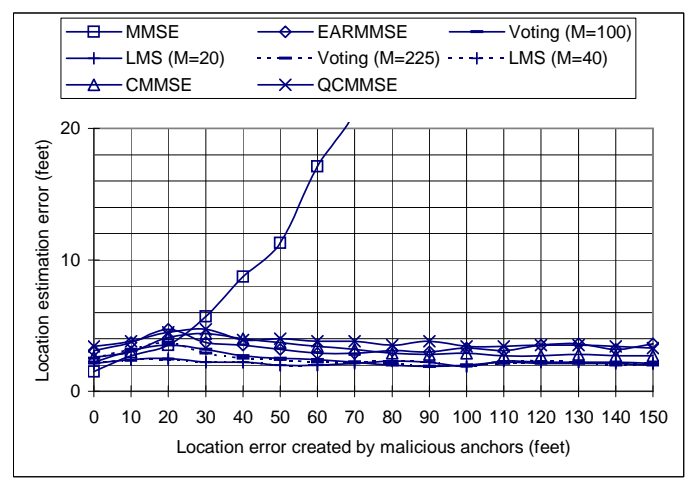

(a) 1 malicious anchor

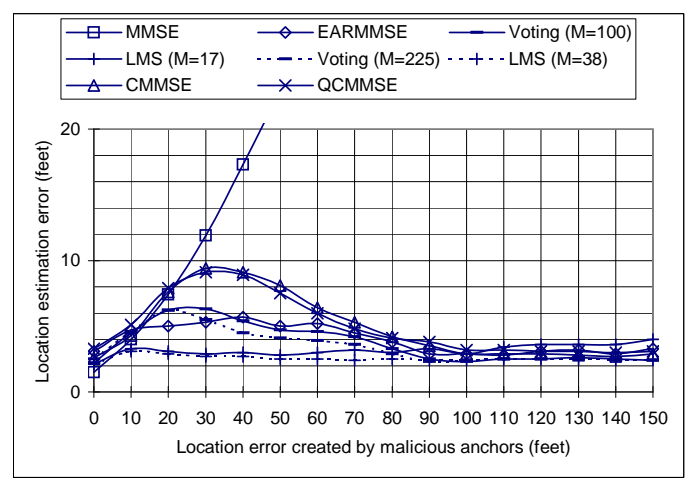

(c) 4 colluding malicious anchors

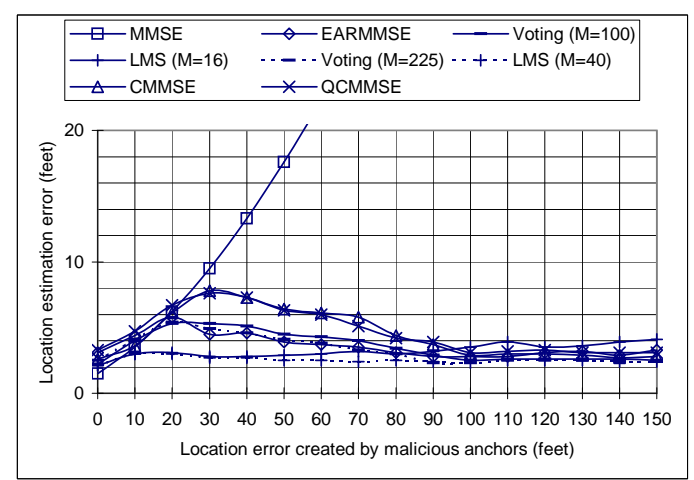

(b) 4 non-colluding malicious anchors

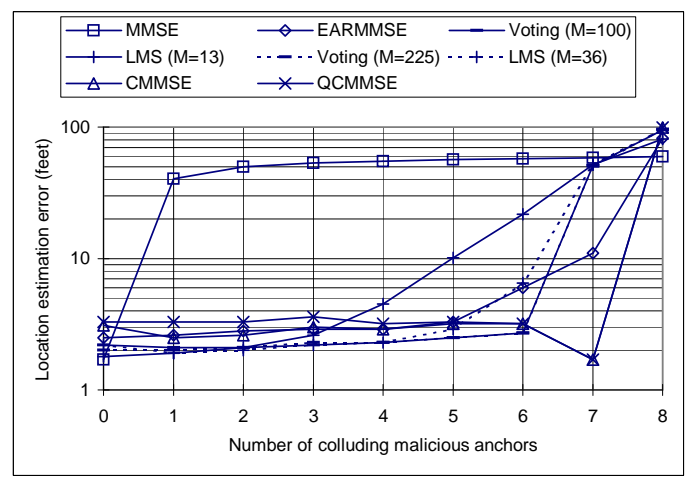

(d) varying number of colluding malicious anchors (error created by malicious anchors is 100 feet)

Figure 3: Location estimation error in different scenarios in field experiments

Under each attack scenario, we investigate the resiliency of CMMSE and QCMMSE compared with the previous approaches in terms of localization error and malicious location reference detection rate, and the algorithm efficiency in terms of execution time. In the experiment we vary the error injected from 10 to 150 feet with a 10 feet increment. In all scenarios, we run each scheme 10 rounds for each random placement of malicious anchors. We then compute the average location estimation error and execution time for each scheme.

From Figures 3(a), 3(b), 3(c), and 3(d), all schemes except for the basic MMSE method have bounded location estimation error, and they can tolerate not only non-colluding malicious anchors but also non-majority colluding malicious anchors. The proposed CMMSE and QCMMSE schemes have slightly higher location estimation errors than EARMMSE, voting-based and LMS schemes; however, the location estimation errors in CMMSE and QCMMSE schemes are in general comparable with the other alternatives. As shown in Figure 3(d), both CMMSE and QCMMSE in fact has smaller location estimation errors than the LMS scheme when the number of colluding malicious anchors is large.

To support our explanation, we investigate the effectiveness of each scheme to filter out malicious location references under different amounts of error injection. For each scheme in each attack scenario, we capture the number of malicious location references that have been successfully identified in each round and calculate the average detection rate over 10 rounds. Figures 4(a), 4(b), and 4(c) show the success rate of removing malicious location references in our experiments. As we can see, all schemes, including the proposed CMMSE and QCMMSE, have similar results in these figures.

All the schemes under investigation fail to identify and remove malicious location references when the injected errors are small $(<70$ feet). When the injected error is at 10 feet, no scheme is able to identify and remove the malicious location references. This is because the malicious anchors behave in a way very similar to benign anchors, and the injected errors are indistinguishable from normal measurement errors. In such cases, the errors introduced by malicious anchors do not introduce significant impact on location estimation.

Figure 4(d) provides the results on the malicious location reference detection probability when we have $1 \sim 8$ ma- 


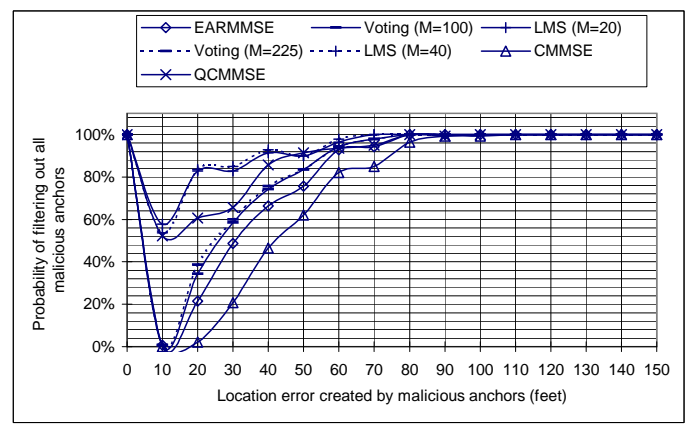

(a) 1 malicious anchor

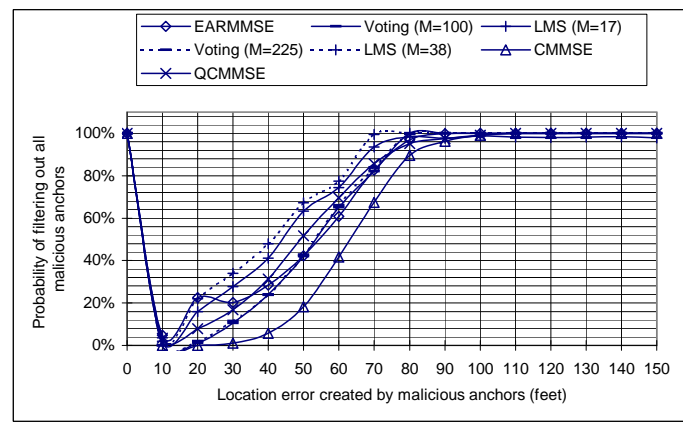

(c) 4 colluding malicious anchors



(b) 4 non-colluding malicious anchors

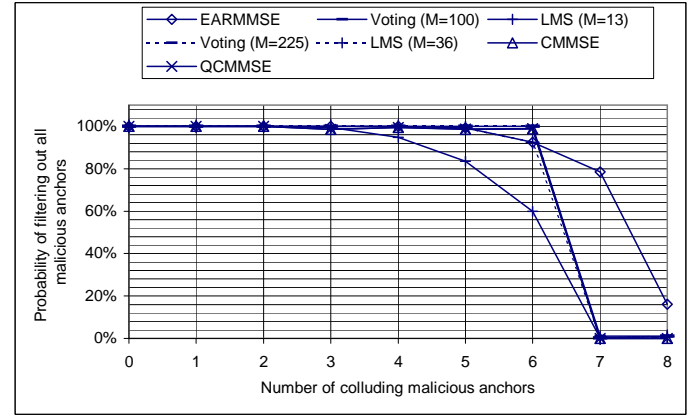

(d) varying number of colluding malicious anchors (error created by malicious anchors is 100 feet)

Figure 4: Success rate of removing malicious location references in field experiments

licious location references. Since the injected error is 100 feet, all schemes should be able to identify and remove the malicious location references when the number of colluding ones is small (1 6). The figure shows that the LMS scheme is the first scheme to break down, and the EARMMSE method provides the best detection rate. Despite the observable differences in Figure 4(d), all the schemes have very similar results.

The above results indicate that the proposed CMMSE and QCMMSE schemes have slightly worse but comparable performance in terms of location estimation errors compared with the EARMMSE, the voting-based, and the LMS schemes. In the remaining discussion for field experiments, we focus on the efficiency of the schemes under investigation.

Figure 5 shows the execution time of the schemes under investigation on MICAz motes. In all the four evaluation scenarios, we can see the execution time of the voting-based and the LMS schemes are much more expensive than the other schemes. EARMMSE and CMMSE have similar execution time in all scenarios, and are much more efficient than the voting-based and the LMS schemes, but observably less efficient than QCMMSE. In most cases, QCMMSE is at least twice as fast as EARMMSE. The most efficient one in Figure 5 is the basic MMSE scheme, which is included as a reference. Unfortunately, the basic MMSE scheme is not resilient to malicious anchors at all.

Combining the results obtained in the field experiments, we can see that the proposed CMMSE and QCMMSE schemes have slightly worse but comparable location estimation errors compared with the alternative schemes, but are in general much more efficient in terms of computation. In particular, QCMMSE requires the least computation among all the resilient schemes while maintaining a similar level of resiliency against malicious anchors.

\subsection{Simulation}

A limitation of field experiments is that we cannot obtain comprehensive evaluation results through a large number of random deployments. To get better understanding of the performance results, we also perform simulation experiments aimed at verifying and confirming the conclusion drawn from the field experiments. Since the simulation is executed in PC rather than motes, we focus on our evaluation on location estimation errors.

In all simulations, we use the same parameters for the target field, signal range, and maximum measurement error 


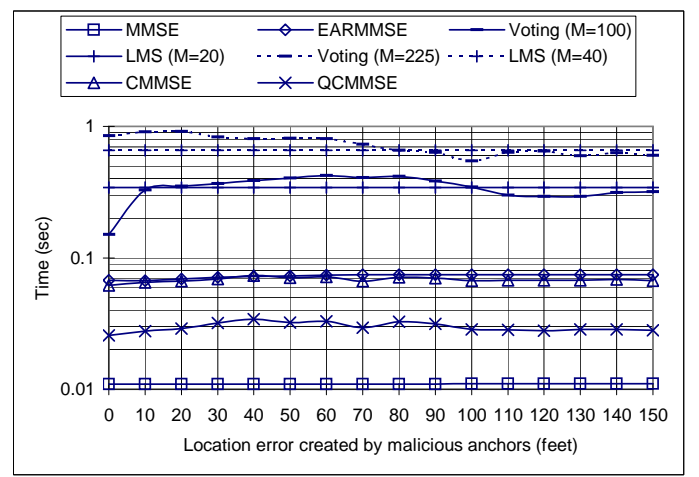

(a) 1 malicious anchor

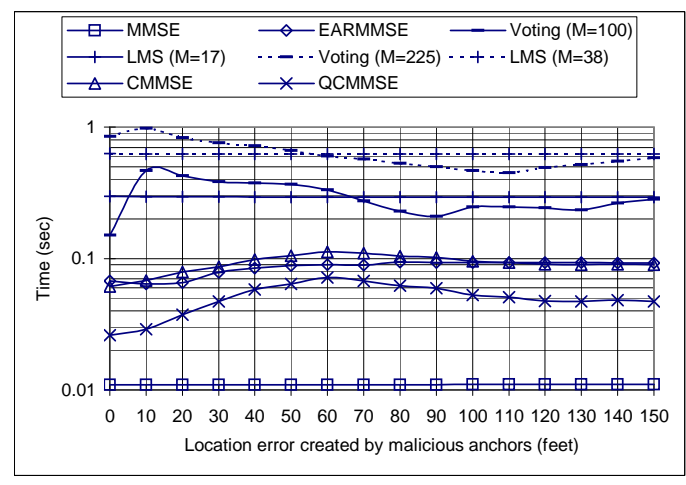

(c) 4 colluding malicious anchors

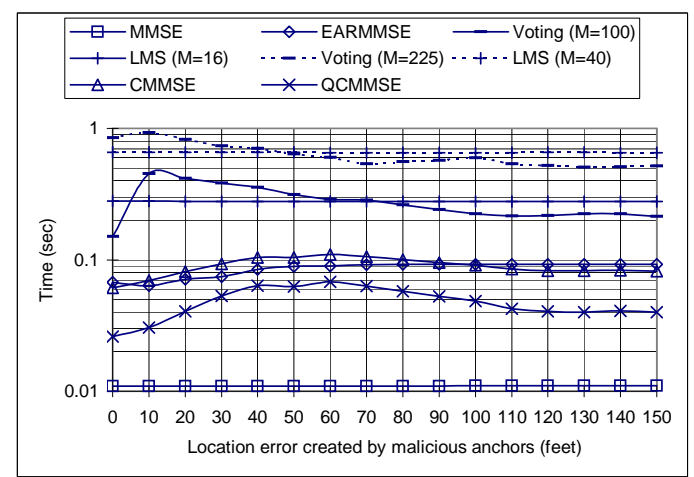

(b) 4 non-colluding malicious anchors

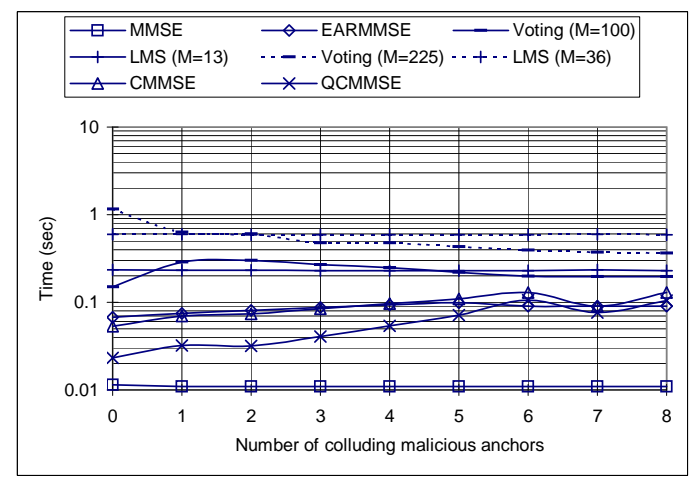

(d) varying number of colluding malicious anchors (error created by malicious anchors is 100 feet)

Figure 5: Execution time in different scenarios in field experiments

as in the field experiments. For simplicity, we simulate the distance measurement error using a uniform distribution between $-\epsilon_{\max }$ and $\epsilon_{\max }$. In each simulation, a set of 14 anchors, including both benign and malicious anchors, are deployed in the target field. The non-anchor sensor node, which needs to localize itself, is located at the center of this target field. So we know the true location of this node. We use the same four attack scenarios as in the field experiments. In our evaluation, we run 1,000 rounds of simulation in TOSSIM (the simulator of TinyOS) for each data point to calculate the average location estimation error. In each round we randomize the location of each anchor.

We first use the same deployment of anchor nodes as in the field experiments to verify the results obtained through field experiments. Figure 6 shows the resulting location estimation errors. We can see the location estimation errors are very similar to the results obtained in the field experiments. The difference is because of the simulated measurement errors.

We then use random deployment of the 14 anchors in our simulation. Figure 7 shows the location estimation error for all evaluated schemes in each scenario. We can see that the location estimation errors in all these schemes are consistent with the evaluation results obtained in the field experiments. Thus, we can conclude that the CMMSE and QCMMSE schemes indeed have comparable performance as the alternative secure localization schemes. Further considering the fast execution time of these two schemes, we can see that these two schemes, especially QCMMSE, are suitable for secure and resilient location estimation on resource constrained sensor nodes.

\section{Conclusion}

In this paper, we developed CMMSE and its variation QCMMSE to efficiently tolerate attacks against localization in WSN. Through random seed selection and triplet examination, CMMSE and QCMMSE use the basic MMSE method to filter out malicious location references efficiently. The algorithm is well suited for the current generation of low end wireless sensor nodes. To evaluate the proposed schemes, we implemented CMMSE, QCMMSE, and all the recent 


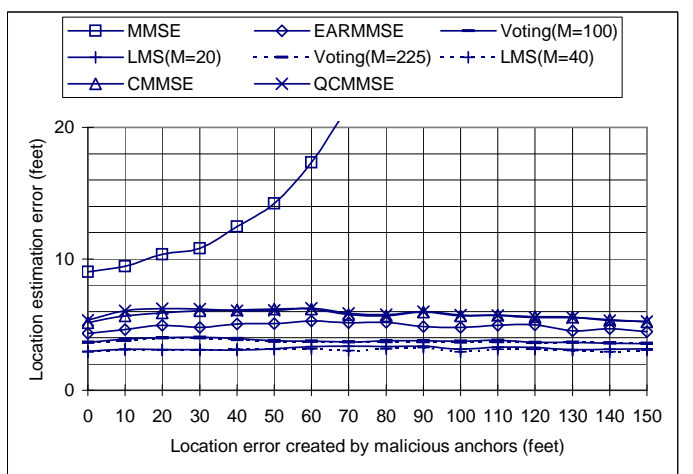

(a) 1 malicious anchor

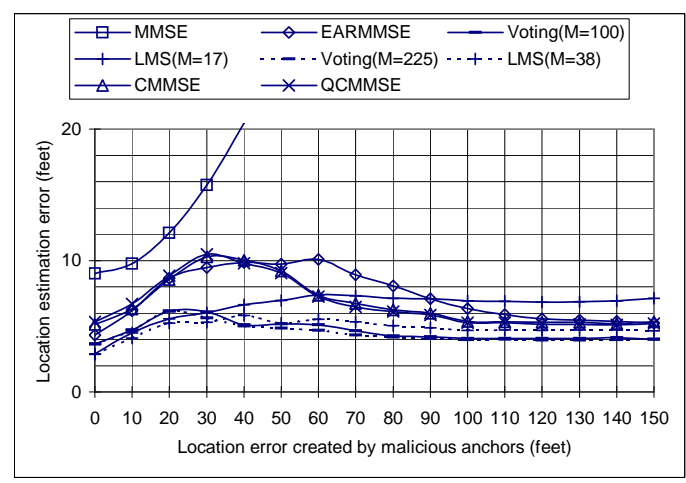

(c) 4 colluding malicious anchors

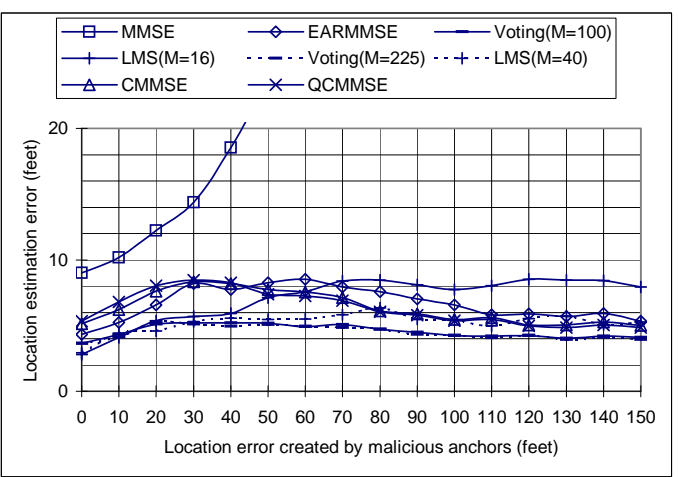

(b) 4 non-colluding malicious anchors

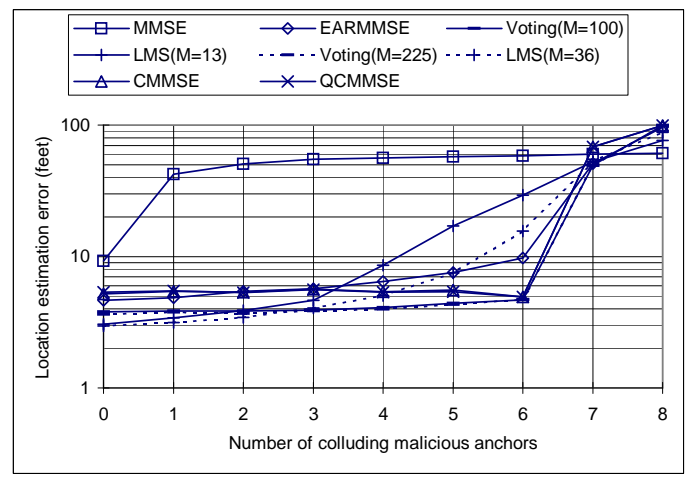

(d) varying number of colluding malicious anchors (error created by malicious anchors is 100 feet)

Figure 6: Location estimation error in simulation (same anchor location as field experiments)

secure and resilient localization schemes that can be used on the current generation of sensor platforms, including EARMMSE [14], the voting-based scheme [15], and the LMS-based scheme [13]. We have performed thorough experimental evaluation through both outdoor field experiments and simulation. Our results conclude that the newly proposed schemes, particularly QCMMSE, has the fastest execution among all the resilient localization schemes, and at the same time provide a comparable degree of resiliency against malicious attacks.

All the existing secure and resilient localization schemes, including CMMSE and QCMMSE, require a majority of benign location references. In the future, we will investigate resilient and/or detection techniques complementary to resilient estimation, so that we can still provide resilient location estimation even when the colluding malicious anchors form the majority.

\section{References}

[1] N. Bulusu, J. Heidemann, and D. Estrin. GPS-less low cost outdoor localization for very small devices. In IEEE Personal Communications Magazine, pages 28-34, October 2000.

[2] S. Capkun and J. Hubaux. Secure positioning of wireless devices with application to sensor networks. In Proceedings of IEEE InfoCom'05 (to appear), 2005.

[3] H. Chan, A. Perrig, and D. Song. Random key predistribution schemes for sensor networks. In IEEE Symposium on Research in Security and Privacy, pages 197-213, 2003.

[4] L. Doherty, K. S. Pister, and L. E. Ghaoui. Convex optimization methods for sensor node position estimation. In Proceedings of INFOCOM'01, 2001. 


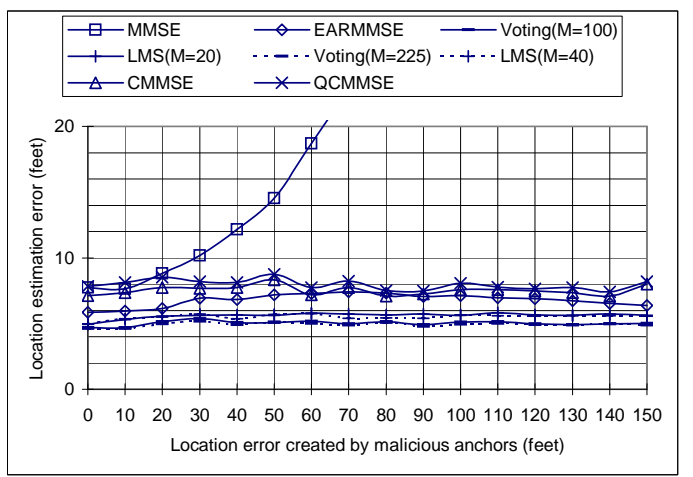

(a) 1 malicious anchor

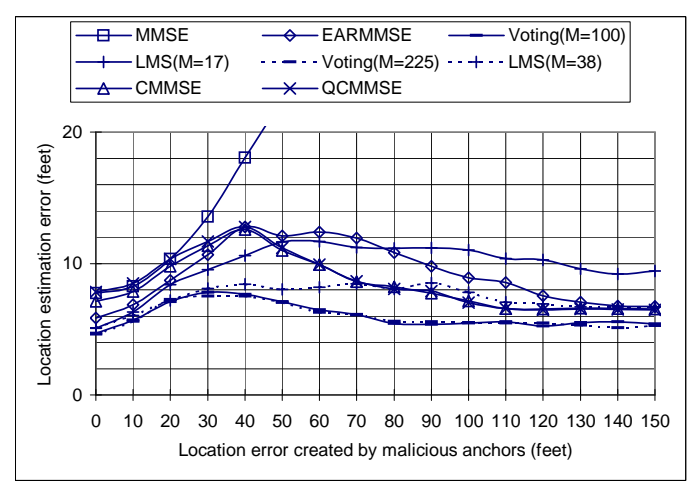

(c) 4 colluding malicious anchors

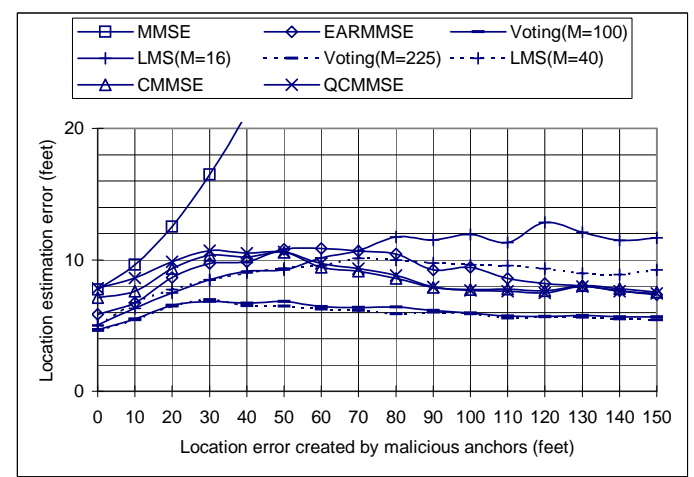

(b) 4 non-colluding malicious anchors

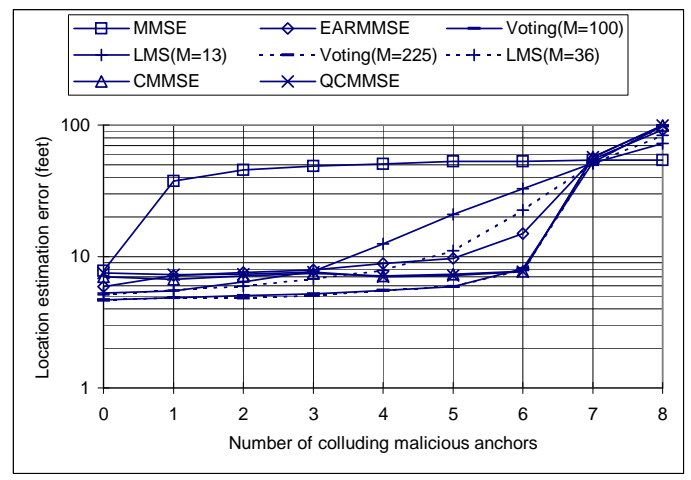

(d) varying number of colluding malicious anchors (error created by malicious anchors is 100 feet)

Figure 7: Location estimation error in simulation (random anchor location)

[5] T. He, C. Huang, B. M. Blum, J. A. Stankovic, and T. F. Abdelzaher. Range-free localization schemes in large scale sensor networks. In Proceedings of ACM MobiCom 2003, 2003.

[6] Y. Hu, A. Perrig, and D. Johnson. Packet leashes: A defense against wormhole attacks in wireless ad hoc networks. In Proceedings of INFOCOM 2003, April 2003.

[7] Y. Hu, A. Perrig, and D. B. Johnson. Wormhole detection in wireless ad hoc networks. Technical Report TR01384, Department of Computer Science, Rice University, Dec 2001.

[8] P. Juang, H. Oki, Y. Wang, M. Martonosi, L. Peh, and D. Rubenstein. Energy-efficient computing for wildlife tracking: Design tradeoffs and early experiences with zebranet. In Proceedings of Tenth International Conference on Architectural Support for Programming Languages and Operating Systems (ASPLOS-X), pages 96-107, October 2002.

[9] C. Karlof, N. Sastry, and D. Wagner. TinySec: A link layer security architecture for wireless sensor networks. In Proceedings of the 2nd ACM Conference on Embedded Networked Sensor Systems (SensSys 2004), November 2004.

[10] B. Karp and H. T. Kung. GPSR: Greedy perimeter stateless routing for wireless networks. In Proceedings of ACM MobiCom 2000, 2000.

[11] L. Lazos, S. Capkun, and R. Poovendran. Rope: Robust position estimation in wireless sensor networks. In Proceedings of the Fourth International Conference on Information Processing in Sensor Networks (IPSN '05), April 2005.

[12] L. Lazos and R. Poovendran. Serloc: Robust localization for wireless sensor networks. ACM Transactions on Sensor Networks, 1(1):73-100, August 2005. 
[13] Z. Li, W. Trappe, Y. Zhang, and B. Nath. Robust statistical methods for securing wireless localization in sensor networks. In Proceedings of the Fourth International Conference on Information Processing in Sensor Networks (IPSN '05), April 2005.

[14] D. Liu, P. Ning, and W. Du. Attack-resistant location estimation in wireless sensor networks. Technical Report TR-2004-29, North Carolina State University, Department of Computer Science, 2004. Revised August 2005.

[15] D. Liu, P. Ning, and W. Du. Attack-resistant location estimation in wireless sensor networks. In Proceedings of the Fourth International Conference on Information Processing in Sensor Networks (IPSN '05), April 2005.

[16] D. Liu, P. Ning, and R. Li. TinyKeyMan: Key management for sensor networks. http: / / discovery . csc . ncsu.edu/software/TinyKeyMan/.

[17] D. Liu, P. Ning, and R. Li. Establishing pairwise keys in distributed sensor networks. ACM Transactions on Information and System Security (TISSEC), 8(1):41-77, February 2005.

[18] R. Nagpal, H. Shrobe, and J. Bachrach. Organizing a global coordinate system from local information on an ad hoc sensor network. In IPSN'03, 2003.

[19] A. Nasipuri and K. Li. A directionality based location discovery scheme for wireless sensor networks. In Proceedings of ACM WSNA'02, pages 105-111, September 2002.

[20] J. Newsome, R. Shi, D. Song, and A. Perrig. The sybil attack in sensor networks: Analysis and defenses. In Proceedings of IEEE International Conference on Information Processing in Sensor Networks (IPSN 2004), April 2004.

[21] J. Newsome and D. Song. GEM: graph embedding for routing and data-centric storage in sensor networks without geographic information. In Proceedings of the First ACM Conference on Embedded Networked Sensor Systems (SenSys '03), pages 76-88, Nov 2003.

[22] D. Niculescu and B. Nath. Ad hoc positioning system (APS) using AoA. In Proceedings of IEEE INFOCOM 2003, pages 1734-1743, April 2003.

[23] D. Niculescu and B. Nath. DV based positioning in ad hoc networks. In Journal of Telecommunication Systems, 2003.

[24] B. Parno, A. Perrig, and V. Gligor. Distributed detection of node replication attacks in sensor networks. In IEEE Symposium on Security and Privacy, May 2005.

[25] S. Ratnasamy, B. Karp, L. Yin, F. Yu, D. Estrin, R. Govindan, and S. Shenker. GHT: A geographic hash table for data-centric storage. In Proceedings of 1st ACM International Workshop on Wireless Sensor Networks and Applications, Sep 2002.

[26] A. Savvides, C. Han, and M. Srivastava. Dynamic fine-grained localization in ad-hoc networks of sensors. In Proceedings of ACM MobiCom '01, pages 166-179, July 2001.

[27] A. Savvides, H. Park, and M. Srivastava. The bits and flops of the n-hop multilateration primitive for node localization problems. In Proceedings of ACM WSNA '02, pages 112-121, September 2002.

[28] B. H. Wellenhoff, H. Lichtenegger, and J. Collins. Global Positions System: Theory and Practice. Springer Verlag, 4th edition, 1997.

[29] Y. Yu, R. Govindan, and D. Estrin. Geographical and energy aware routing: A recursive data dissemination protocol for wireless sensor networks. Technical Report UCLA/CSD-TR-01-0023, UCLA, Department of Computer Science, May 2001. 